\title{
Early-type galaxies in the Antlia cluster: catalogue and isophotal analysis
}

\author{
Juan P. Calderón, ${ }^{1,2,3 \star}$ Lilia P. Bassino, ${ }^{1,2,3}$ Sergio A. Cellone ${ }^{1,3,4}$ and Matías Gómez ${ }^{5}$ \\ ${ }^{1}$ Consejo Nacional de Investigaciones Científicas y Técnicas, Rivadavia 1917, Buenos Aires, Argentina \\ ${ }^{2}$ Instituto de Astrofísica de La Plata (CCT La Plata - CONICET - UNLP), La Plata, Argentina \\ ${ }^{3}$ Facultad de Ciencias Astronómicas y Geofísicas, Universidad Nacional de La Plata, Paseo del Bosque, B1900FWA La Plata, Argentina \\ ${ }^{4}$ Complejo Astronómico El Leoncito (CONICET - UNLP - UNC - UNSJ), San Juan, Argentina \\ ${ }^{5}$ Departamento de Ciencias Físicas, Facultad de Ciencias Exactas, Universidad Andres Bello, Santiago, Chile
}

Accepted 2018 February 26. Received 2018 February 26; in original form 2017 December 14

\begin{abstract}
We present a statistical isophotal analysis of 138 early-type galaxies in the Antlia cluster, located at a distance of $\sim 35 \mathrm{Mpc}$. The observational material consists of CCD images of four $36 \times 36 \operatorname{arcmin}^{2}$ fields obtained with the MOSAIC II camera at the Blanco 4-m telescope at Cerro Tololo Interamerican Observatory. Our present work supersedes previous Antlia studies in the sense that the covered area is four times larger, the limiting magnitude is $M_{B} \sim-9.6 \mathrm{mag}$, and the surface photometry parameters of each galaxy are derived from Sérsic model fits extrapolated to infinity. In a companion previous study we focused on the scaling relations obtained by means of surface photometry, and now we present the data, on which the previous paper is based, the parameters of the isophotal fits as well as an isophotal analysis. For each galaxy, we derive isophotal shape parameters along the semimajor axis and search for correlations within different radial bins. Through extensive statistical tests, we also analyse the behaviour of these values against photometric and global parameters of the galaxies themselves. While some galaxies do display radial gradients in their ellipticity $(\epsilon)$ and/or their Fourier coefficients, differences in mean values between adjacent regions are not statistically significant. Regarding Fourier coefficients, dwarf galaxies usually display gradients between all adjacent regions, while non-dwarfs tend to show this behaviour just between the two outermost regions. Globally, there is no obvious correlation between Fourier coefficients and luminosity for the whole magnitude range $\left(-12 \gtrsim M_{V} \gtrsim-22\right)$; however, dwarfs display much higher dispersions at all radii.
\end{abstract}

Key words: galaxies: clusters: general-galaxies: clusters: individual: Antlia-galaxies: $\mathrm{dwarf}$-galaxies: elliptical and lenticular, $\mathrm{cD}$-galaxies: fundamental parameters.

\section{INTRODUCTION}

Since the early work of Sérsic (1968), the study of the surface brightness profiles of elliptical galaxies (E) has reached a state in which peculiarities are more the rule than the exception. Even long-considered 'canonical' examples of purely elliptical shape like NGC 3379 (see e.g. Statler 1994) are nowadays understood as prime focus for isophote twisting, large shells and arcs and complex structure extending many effective radii; these evidence cast serious doubts on the existence of alleged pure $\mathrm{E}$ as a class.

Even for E galaxies with symmetrical isophotes, there is usually extra light that distorts the profile (e.g. Malin \& Carter 1983; Schweizer \& Seitzer 1988; Seitzer \& Schweizer 1990; Barnes \& Hernquist 1992). Thus, in many cases, the isophotes of these galax- ies deviate systematically from pure ellipses. Depending on the shape of those deviations, they are referred to as 'discy' or 'boxy' isophotes. Discy isophotes are the consequence of light excesses along the main axes (major and minor) with respect to a perfectly elliptical, while boxy isophotes are the consequence of deformations along directions at $45^{\circ}$ from the main axes. In fact, galaxies within these two types of isophote classifications present quite different characteristics, defining two 'families'. Boxy early-type galaxies (ETGs) are usually luminous and massive, have significant radio and X-ray emission, have 'core' nuclear profiles and slow rotation; discy ETGs, in turn, tend to be fainter, have significant rotation, and no (or faint) X-ray or radio activity (Ferrarese et al. 1994; van den Bosch et al. 1994; Rest et al. 2001; Lauer et al. 2005).

The analysis of possible correlations between isophotal shapes and other parameters that characterize the isophotes, or the properties of the galaxies themselves, has been the subject of many studies. Bender et al. (1989) and Nieto \& Bender (1989), two seminal papers 
on the subject, performed detailed studies of the shapes of isophotes of massive E galaxies, and concluded that there is no strong correlation with any photometric parameter like effective radius or surface brightness. More recently, Krajnović et al. (2013) analysed the nuclear slope of 135 ETGs and found no evidence of bimodality regarding boxy or discy isophotes. Using the integral-field spectroscopy obtained by the ATLAS ${ }^{3 \mathrm{D}}$ survey, Emsellem et al. (2011) also pointed out that the $a_{4} / a$ parameter, i.e. the Fourier coefficient that defines 'disciness/boxiness', is not directly related with any kinematic properties in their sample of 260 ETGs. However, galaxies surrounded by X-ray haloes have generally irregular or boxytype isophotes. Bender et al. (1989) found that boxy galaxies have higher mass-luminosity ratios $\left(M / L \sim 11.5 \pm 0.9 \mathrm{M}_{\odot} / \mathrm{L}_{\odot}\right)$ than discy-type galaxies $\left(M / L \sim 6.4 \pm 0.6 \mathrm{M}_{\odot} / \mathrm{L}_{\odot}\right)$. Regarding galaxy luminosity, the fainter galaxies tend to be discy, while those with higher luminosities tend to be boxy. These observed correlations mark the cause of the dichotomy between the isophote shapes and its relation with galaxy formation history (Bekki \& Shioya 1997). Also, there is growing evidence of a correlation between the age and the shape of galaxies, in the sense that core Es have older stellar populations than power-law ones (Ryden, Forbes \& Terlevich 2001). In addition, He, Hao \& Xia (2014) investigated the relationships among isophotal shapes, galaxy brightness profile, and kinematic properties of a sample of ETGs from DSS Data Release 8 with kinematic properties available from the ATLAS ${ }^{3 \mathrm{D}}$ survey. They found no clear relation between the Sérsic index and isophotal shape. Instead, they found correlations between the Fourier coefficient $a_{4} / a$, ellipticity, and specific angular momentum $\lambda r_{\mathrm{e}} / 2$ for power-law galaxies, while no relation was found for core ETGs.

From the theoretical side, there have been many attempts to understand the origin of discy and boxy Es. Naab, Khochfar \& Burkert (2006, and references therein) used semi-analytical simulations to conclude that discy Es are mainly produced by non-equal mergers of two disc galaxies, while equal-mass mergers tend to produce boxy Es. In addition, Khochfar \& Burkert (2005) concluded that the isophotal shapes of merger remnants also depend on the morphology of their progenitors and the subsequent gas infall.

Our present study focuses on the Antlia cluster, which is recognized as the third nearest rich galaxy cluster, after Fornax and Virgo. Its galaxy population ranges in luminosity between -12 and -22 mag in the $T_{1}$ band, while no study of the relationship between their isophotes and global parameters has still been done. The first study of its galaxy content was performed by Ferguson \& Sandage (1990), who constructed the photographic catalogue FS90. On the basis of CCD images, a deeper analysis of the ETGs located at the central zone of Antlia was performed (Smith Castelli et al. 2008a,b, 2012). In the present work, we extend the studied region approximately four times, determining total (not isophotal) magnitudes and colours. Structural parameters have also been obtained by means of Sérsic model fits. Half of the studied galaxies are included in the FS90 catalogue and the rest, mostly in the fainter regime, are new ones. The total sample amounts to 138 ETGs, 59 of them being spectroscopically confirmed Antlia members. These data have already been used in a previous companion paper (Calderón et al. 2015) to study the Antlia galaxies scaling relations.

This paper presents the catalogue of structural parameters of ETGs in the Antlia cluster and, on the basis of these data, an isophotal analysis of the galaxy sample is made. The paper is organized in the following way: in Section 2 we describe the imaging data reduction, while the galaxy sample selection is briefly presented in Section 3. Section 4 presents the computation of the geometrical parameters, while in Section 5 we describe the surface photometry

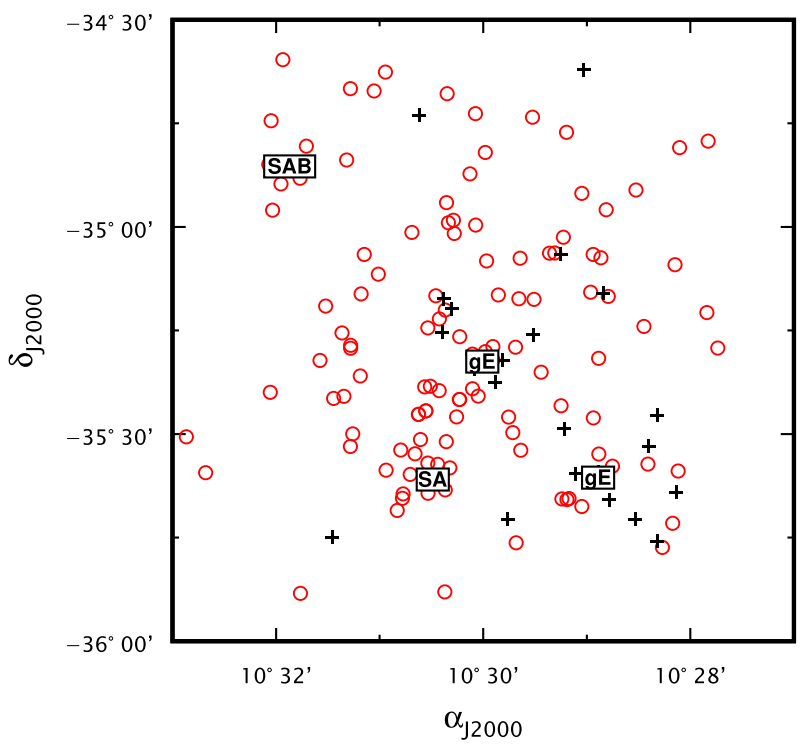

Figure 1. Projected distribution of all galaxies in the sample. The faintest galaxies in the sample ( $\mathrm{dE}$ and $\mathrm{dSph})$ are indicated with red circles, and the brightest ones with black crosses. Four of the most luminous galaxies in the cluster are labelled with their morphologies: NGC 3268 (' $\mathrm{gE}$ ', at the centre of the figure), NGC 3258 ('gE', at south-west), NGC 3281 ('SAB'), and NGC 3273 ('SA'). North is up and east to the left.

method used to obtain each galaxy profile. Our results are presented in Section 6, and we discuss them in Section 7. The main conclusions are contained in Section 8. The full catalogue is available in electronic format.

\section{DATA}

The photometric data used in this paper are CCD images obtained with the MOSAIC II camera, mounted on the Victor Blanco 4-m telescope at the Cerro Tololo Interamerican Observatory (CTIO, Chile). We used the Kron-Cousins $R$ and Washington $C$ filters (Canterna 1976). The $R$ filter was chosen instead of the original Washington $T_{1}$ because of its better efficiency (Geisler 1996), while just a small change of zero-point $\left(R-T_{1}=0.02\right)$ is needed to transform between them (Dirsch, Richtler \& Bassino 2003). Each image covers $36 \times 36 \operatorname{arcmin}^{2}$, which corresponds to about $370 \times 370 \mathrm{kpc}^{2}$ according to the adopted Antlia distance (Dirsch et al. 2003, $d=35 \mathrm{Mpc} ; m-M=32.73$ ). The MOSAIC II camera had a resolution of 0.27 arcsec pixel ${ }^{-1}$ and was constituted by eight CCDs. In order to erase the gaps between the CCDs, it is necessary to take a series of slightly shifted exposures (dithering) and then combine them. Fig. 1 shows the projected spatial distribution of the four MOSAIC fields used in this work, in the $R$ band. Red circles represent the faintest galaxies in the sample ( $\mathrm{dE}$ and $\mathrm{dSph}$ ), while black crosses indicate the brightest ones. We also added the location of the more luminous galaxies in the sample: NGC 3258, NGC 3268, NGC 3281, and NGC 3273. We have already described the images in Calderón et al. (2015), as well as the calibration to the standard system and the resulting signal-to-noise ratio $(\mathrm{S} / \mathrm{N}$; see Section 5.3) of the brightness profiles, which is extremely relevant to low surface-brightness galaxies. As a consequence, we briefly highlight here the most important steps of images' reduction, as they may be of interest.

The MOSAIC II images reduction was made using the MSCRED package within IRAF, which has been written specially for data of 
similar characteristics (Valdes 1997). The first step consisted in running the task CCDPROC on all the images, in order to perform the basic calibration (overscan subtraction, trimming, bad pixel replacement, zero level subtraction, and flat-fielding). As we are using images with a large field of view (FOV), it is necessary to have an accurate celestial coordinate system. Then, to correct the astrometric solution we ran the MSCCMATCH task, which uses a list of reference celestial coordinates of stars located in the field, to match against the same objects on the MOSAIC images. A polynomial relation between the observed positions and the reference coordinates is obtained. This relation may include a zero-point shift, a scale change, and axis rotation for both coordinate axes. Next, the fit was applied to the multi-extension images and, using MSCIMAGE, it was possible to get an output image in the correct WCS (World Coordinate System). If any residual large-scale gradients were present in the sky background of individual exposures, they were removed using MSCSKYSUB. In the following step, we used MSCIMATCH to match the intensity scales on the different images to be finally combined into the stacked image. Finally, for each filter and each field, the individual exposures were combined into a single deep one using MSCSTACK.

\section{THE GALAXY SAMPLE}

Our galaxy sample comes from the four MOSAIC-II fields described in the previous section and is composed of 107 Antlia galaxies considered as 'members' and 77 new galaxies not catalogued before (Calderón et al. 2015). The 'member' galaxies were those selected from the FS90 catalogue with membership status 1 ('definite members') plus those which have measured radial velocities in the range of $1200-4200 \mathrm{~km} \mathrm{~s}^{-1}$ (Smith Castelli et al. 2008a). We can select galaxies with membership status 1 from FS90 as 'members' due to the reliability of FS90 morphological membership classification, already quantified in previous works (e.g. Smith Castelli et al. 2012, and references therein). Out of the 77 new galaxies, only 31 can be considered as 'candidates' because they satisfy the following criteria that ensure a reliable early-type morphological classification: they have smooth and continuous profiles with reasonable $\mathrm{S} / \mathrm{N}$ out to the $\sim 27.5 \mathrm{mag} \operatorname{arcsec}^{-2}$ in $R$ band, no obvious spiral structure present in the residuals of the fits, etc. These criteria are fully explained in Calderón et al. (2015). In addition, a photometric criterion was also considered, from the colour-magnitude relation (CMR) for the extended objects in the field: only new galaxies located within $\pm 3 \sigma$ of the CMR of the cluster members were selected. The CMR of ETGs in galaxy clusters is a well-defined, universal relation with very small scatter (e.g. Lisker, Grebel \& Binggeli 2008; Penny \& Conselice 2008; Jaffé et al. 2011; Mei et al. 2012).

Our sample is $\sim 5$ mag deeper than FS90, as the FS90 catalogue is complete to $B_{\mathrm{T}}=18 \mathrm{mag}$, which corresponds to $M_{B}=-14.7 \mathrm{mag}$ at our adopted Antlia distance, while here we reach $M_{\mathrm{T}_{1}} \sim-12 \mathrm{mag}$, which corresponds to $M_{B} \sim-9.6$ mag (Fukugita, Shimasaku \& Ichikawa 1995).

\section{ISOPHOTAL ANALYSIS AND COMPUTATION OF GEOMETRICAL PARAMETERS}

We used the ELLIPSE (Jedrzejewski 1987) task within the ISOPHOTE IRAF's package to obtain the observed surface brightness profiles (surface brightness versus semimajor axis $a$ ). The semimajor axis was transformed into equivalent radius $(r=\sqrt{a b}=a \sqrt{1-\epsilon}, a$ being the isophote semimajor axis and $\epsilon$ the ellipticity) for all ETGs in the sample.

The initial values needed for the Fourier fitting, like the geometric centre, initial ellipticity, and position angle of the first trial ellipse, were estimated by visual inspection, for each galaxy in the sample. The intensity $I(\theta)$ along the trial ellipse is described by a Fourier series,

$I(\theta)=I_{0}+\sum_{n=1}^{N} A_{n} \sin (n \theta)+B_{n} \cos (n \theta)$,

where $I_{0}$, is the mean isophotal intensity along the ellipse, $N$ is the highest harmonic fitted, $\theta$ is the azimuthal angle measured from the major axis, and $A_{n}$ and $B_{n}$ with $n=1,2, \ldots$ are the harmonic amplitudes of the Fourier series. If the isophotes were perfect ellipses (which is not the case for real galaxies), the coefficients with $n \leq 2$ would be the only not null ones. The fit begins with the assumption that the first two orders $\left(A_{1}, A_{2}, B_{1}, B_{2}\right)$ are non-zero. The $A_{n}$ and $B_{n}$ coefficients provided by ELLIPSE are normalized to the semimajor axis $a$ and corrected by the local intensity gradient. The output ellipse coefficients $B_{n}$ are converted to $a_{n} / a$ using

$\frac{a_{n}}{a}=B_{n} \sqrt{1-\epsilon}=B_{n} \sqrt{b / a}$.

Once the parameters are obtained, the procedure continues with the calculation of the third and fourth harmonic coefficients through a least-squares fit. These coefficients $\left(A_{3}, A_{4}, B_{3}\right.$, and $\left.B_{4}\right)$ determine the deviation of the isophote from a perfect ellipse. This procedure is repeated for the next semimajor axis, defined by the variable STEP in ELLIPSE, until it reaches a pre-defined value of the semimajor axis. We used a linear step for each profile. The ellipticity and position angle are not well determined close to the centre due to seeing; this effect will be analysed in Section 5.2.

The geometrical parameters, such as ellipticity and Fourier coefficients, vary along the galactocentric radius of the surface-brightness profile and, as a consequence, we cannot consider a single characteristic value as representative of the entire galaxy. In order to compare these parameters to other global galaxy properties, we choose to estimate a weighted average value for each parameter along different ranges of effective radius $\left(r_{\mathrm{e}}\right)$. We divide each galaxy into four regions: region 1 , between the seeing radius ( $1 \mathrm{arcsec})$ and $1.5 r_{\mathrm{e}}$; region 2 , from 1.5 to $3.0 r_{\mathrm{e}}$; region 3 , from 3.0 to $4.5 r_{\mathrm{e}}$; and region 4 , further than $4.5 r_{\mathrm{e}}$. Following Chaware et al. (2014), we estimate each parameter within each region by means of expressions like the following:

$$
\left\langle\frac{a_{4}}{a}\right\rangle=\frac{\int_{r_{s}}^{1.5 r_{\mathrm{e}}} \frac{a_{4}(r)}{a} I(r)\left[\sigma_{\frac{a 4}{a}}(r)\right]^{-2} \mathrm{~d} r}{\int_{r_{s}}^{1.5 r_{\mathrm{e}}} I(r)\left[\sigma_{\frac{a 4}{a}}(r)\right]^{-2} \mathrm{~d} r},
$$

which represents the mean weighted value of $a_{4} / a$ in region 1 . That is, all the calculated average parameters are weighted by intensity (in counts) and inversely weighted by the corresponding variance. Note that there will be fewer parameters assigned to region 4 because the fitting of the model to the profile is not always reliable in the outer regions. Table 1 shows an example of the geometrical parameters computed for the galaxies in the sample.

\section{SURFACE BRIGHTNESS PROFILES}

Given the large number of galaxies in the sample, and the fact that the reduction procedure applied to obtain the surface-brightness profiles consists of several steps that can be automatized (i.e. trim the original image, estimate sky level around the galaxy, etc.), we 
Table 1. Geometric parameters obtained for the galaxies in the sample. Columns: (1) ID from FS90, (2)-(4) mean values calculated by equation (3) for $\langle\epsilon\rangle,\left\langle a_{4} / a\right\rangle$, and $\left\langle a_{3} / a\right\rangle$ on each radial range 1 to 4 (first to fourth line, when available). The full table is electronically available.

\begin{tabular}{|c|c|c|c|}
\hline $\begin{array}{l}\text { (1) } \\
\text { ID (FS90) }\end{array}$ & $\begin{array}{l}(2) \\
\langle\epsilon\rangle\end{array}$ & $\begin{array}{c}(3) \\
\left\langle a_{4} / a\right\rangle\end{array}$ & $\begin{array}{c}(4) \\
\left\langle a_{3} / a\right\rangle\end{array}$ \\
\hline 70 & $\begin{aligned} 0.272 & \pm 0.051 \\
0.330 & \pm 0.013 \\
0.306 & \pm 0.001 \\
\ldots & \end{aligned}$ & $\begin{aligned} 0.004 & \pm 0.013 \\
-0.001 & \pm 0.017 \\
0.031 & \pm 0.016 \\
\ldots & \end{aligned}$ & $\begin{array}{c}0.003 \pm 0.012 \\
0.049 \pm 0.071 \\
0.027 \pm 0.016 \\
\quad \ldots\end{array}$ \\
\hline 72 & $\begin{array}{l}0.334 \pm 0.062 \\
0.380 \pm 0.001 \\
0.378 \pm 0.001 \\
0.349 \pm 0.005\end{array}$ & $\begin{aligned}-0.004 & \pm 0.003 \\
-0.002 & \pm 0.001 \\
0.001 & \pm 0.009 \\
-0.010 & \pm 0.034\end{aligned}$ & $\begin{aligned}-0.003 & \pm 0.002 \\
0.002 & \pm 0.005 \\
-0.008 & \pm 0.004 \\
0.006 & \pm 0.024\end{aligned}$ \\
\hline 73 & $\begin{aligned} 0.255 & \pm 0.017 \\
0.245 & \pm 0.018 \\
0.257 & \pm 0.004 \\
\ldots & \end{aligned}$ & $\begin{aligned}-0.001 & \pm 0.004 \\
-0.007 & \pm 0.008 \\
0.049 & \pm 0.143 \\
\ldots & \end{aligned}$ & $\begin{aligned}-0.001 & \pm 0.006 \\
-0.005 & \pm 0.015 \\
0.080 & \pm 0.138 \\
\ldots & \end{aligned}$ \\
\hline 78 & $\begin{array}{c}0.122 \pm 0.001 \\
\ldots \\
\ldots \\
\ldots\end{array}$ & $\begin{aligned}-0.012 & \pm 0.051 \\
0.046 & \pm 0.035 \\
\ldots & \\
\ldots & \end{aligned}$ & $\begin{aligned}-0.043 & \pm 0.060 \\
0.044 & \pm 0.035 \\
\ldots & \\
\ldots & \end{aligned}$ \\
\hline 79 & $\begin{array}{l}0.301 \pm 0.090 \\
0.381 \pm 0.004 \\
0.370 \pm 0.003 \\
0.339 \pm 0.005\end{array}$ & $\begin{aligned} 0.00 & \pm 0.001 \\
-0.001 & \pm 0.002 \\
-0.002 & \pm 0.008 \\
0.057 & \pm 0.020\end{aligned}$ & $\begin{aligned}-0.002 & \pm 0.002 \\
0.00 & \pm 0.003 \\
0.008 & \pm 0.004 \\
-0.030 & \pm 0.025\end{aligned}$ \\
\hline
\end{tabular}

developed an IRAF pipeline in order to obtain results in a homogeneous way. In this section, we describe such procedure adopted to obtain the surface-brightness profiles.

The MOSAIC II images have 8800 pixel $\times 8000$ pixel. Although automatic detection software (e.g. SExTRACTOR; Bertin \& Arnouts 1996) can be carefully configured for faint sources identification, as we deal with early-type galaxies in a nearby cluster, we decided to carry on the galaxy detection just by visual inspection, which has been shown to be a very efficient method in such a case. We started by re-identifying the FS90 galaxies and then looked for new galaxies. After each galaxy detection, a subimage of about 500 pixels $\times 500$ pixels $\left(135 \times 135 \operatorname{arcsec}^{2}\right)$, centred on each object, was cut. Due to the large MOSAIC II field, we preferred to estimate the background (sky level) for each galaxy independently, instead of setting the same background level for the whole image. The adopted size of these subimages was large enough to make a good sky estimation. We first calculated an initial value of the sky level taking the 'mode' from several positions around the galaxy, free from other sources, using the IMEXAMINE task. Then, we subtracted that constant intensity from the subimage and, due to the large-scale residual removal applied on the previous reduction process, we found that our method to estimate the sky was appropriate for the brightness level of the sample. Once the calibrated galaxy profile was obtained, we applied an iterative process to perform a second-order correction of the sky level, until the outer part of the integrated flux profile became as flat as possible for large galactocentric distances. Such corrections remained between $\pm 10 \mathrm{ADU}$ (i.e. less than 5 percent of the mean sky level).

The last step before performing the fit of the model profile was to build a mask for each subimage to remove any objects that might have affected the brightness profile, like foreground stars and cosmetics. In this way, we obtained one mask for each subimage and each filter, using the BADPIXIMAGE task. We also took into account objects hidden in the galaxy brightness, using different display levels. As a consequence, the final masks resulted more accurate than those generated directly by the ELLIPSE task.
Afterwards, we performed a first run of the ELLIPSE task, leaving all the geometric parameters 'free', just to obtain approximate values for the following initial geometric parameters:

(i) $\mathrm{X} 0, \mathrm{y} 0$ : coordinates of the initial isophote centre.

(ii) PA0: initial position angle.

(iii) ELLIP0: initial ellipticity.

(iv) SMA0: initial semimajor axis length.

(v) MAXSMA: maximum semimajor axis length.

For each galaxy, we defined a set of initial parameters in such a way to improve the stability of the isophotal fit. The minimum semimajor axis (MINSMA) was taken as small as possible to be able to fit the very central region of the galaxy. As the images were skysubtracted, we defined the value of MAXSMA as that for which the galaxy brightness approaches zero level. This procedure was applied on the $R$ images as they are deeper than the $C$ ones (Calderón et al. 2015 ). The $R$-band output table was later used as input to ELLIPSE on the $C$-band images to perform the photometry.

If the image had defects that could complicate the fit, and/or the galaxy was so faint that the brightness profile was strongly dependent on the choice of the initial parameters, we kept one of them fixed to allow for a better solution with less degrees of freedom. These galaxies were mainly the faintest dwarf ellipticals (dE) or dwarf spheroidals (dSph). Fixing one or more parameters in the iteration does not modify the total magnitude of the galaxy although information on geometrical parameters may be lost.

Fig. 2 shows examples of the galaxy brightness profiles of two galaxies in the sample (FS90 211 on the left and FS90 307 on the right). From top to bottom, the figure presents the run along $r$ of: $a_{4} / a, \epsilon$, surface brightness $\mu_{\mathrm{T}_{1}}$ (filled circles) along with the fitted Sérsic model (continuous line), and the corresponding residual between model and observed profile. Finally, the $T_{1}$-band image is shown. 


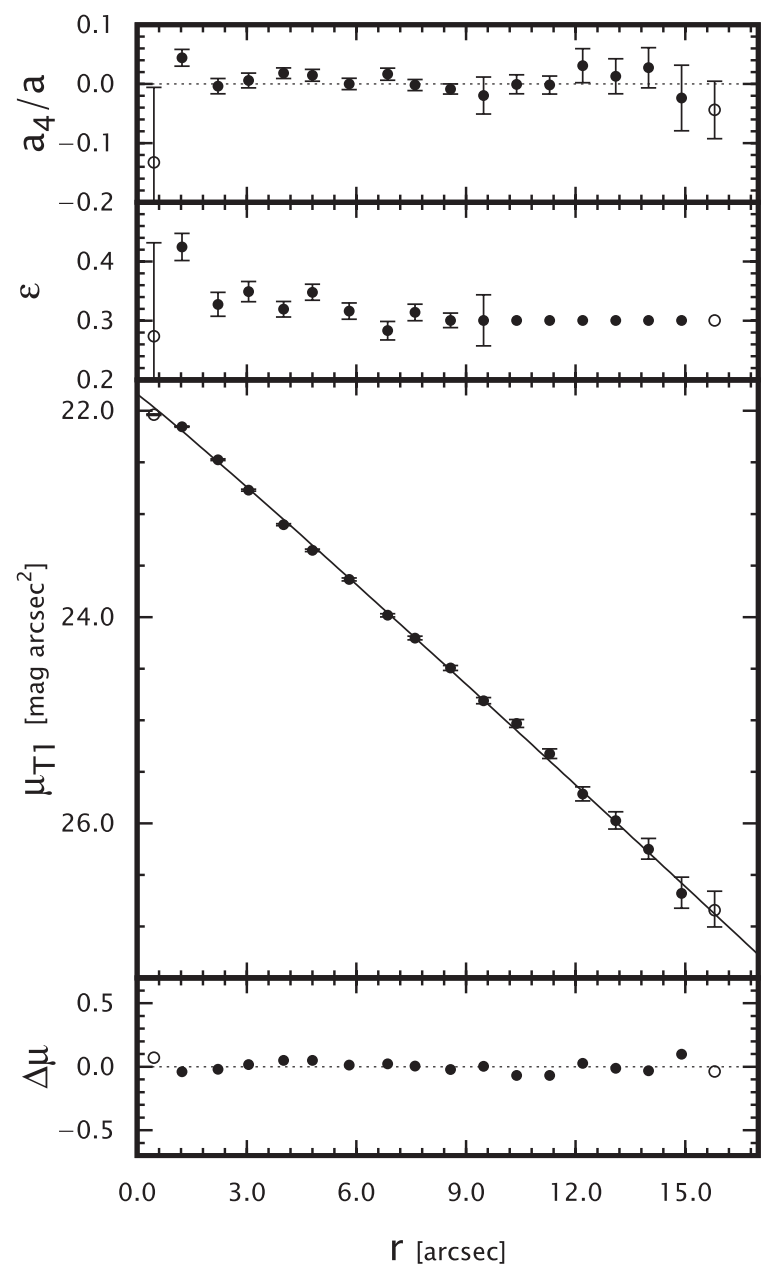

(a)

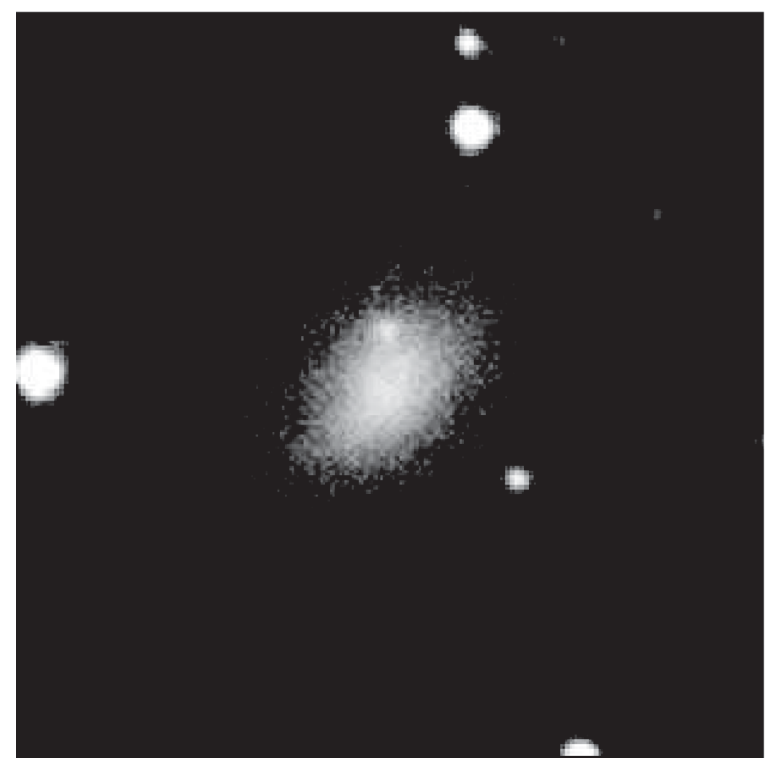

(c)

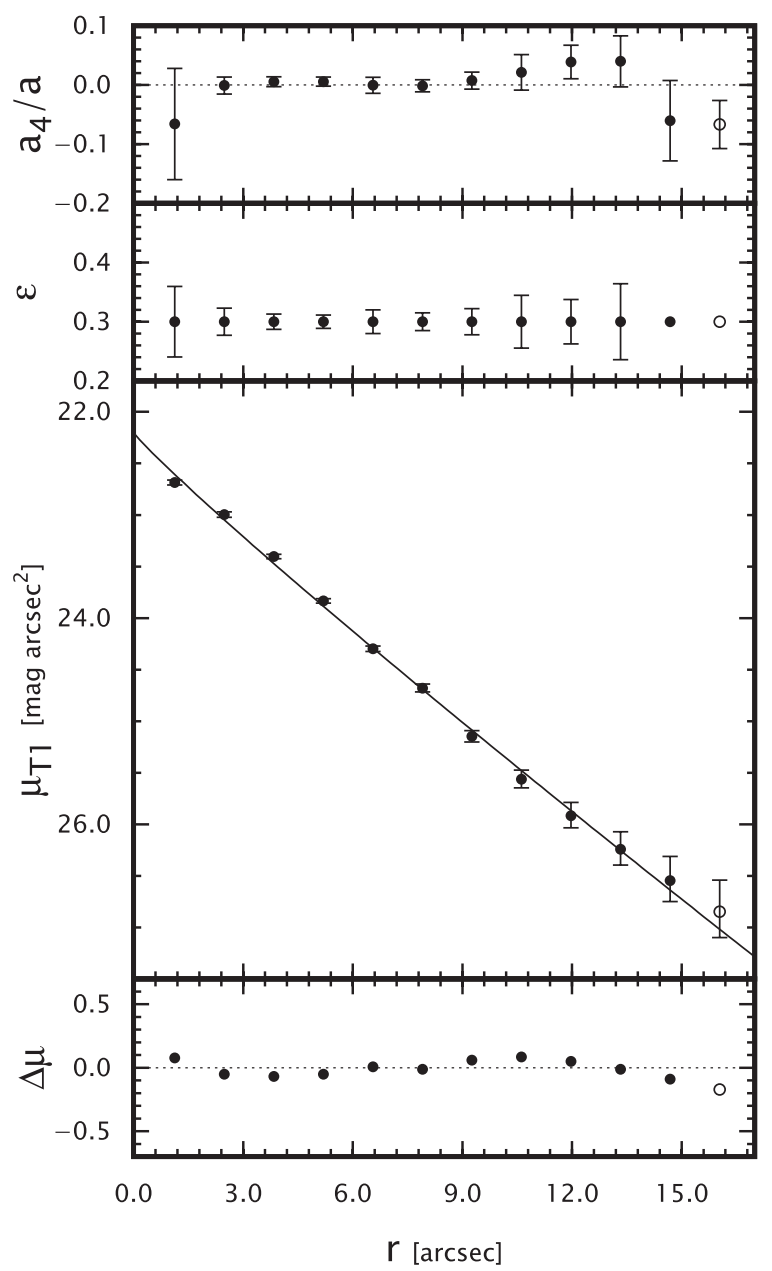

(b)

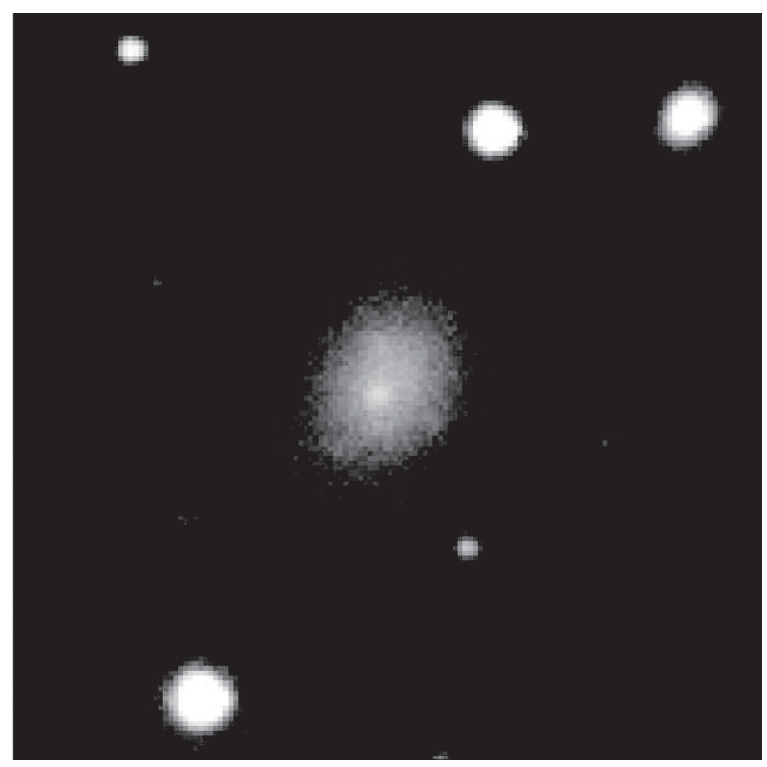

(d)

Figure 2. Two examples of the profile fits on the $T_{1}$-band images: FS90 211 (left) and FS90 307 (right). Parts (a) and (b) show (from top to bottom) the variation along $r$ of $a_{4} / a$, ellipticity $(\epsilon)$, surface brightness $\left(\mu_{\mathrm{T} 1}\right)$, and the residual between the model and the observed profile. On each panel, we show with filled circles the data used in the fits and with open circles the discarded data, all of them with their respective error bars. Parts (c) and (d) show $T_{1}$-band images of each galaxy $(40 \times 40 \operatorname{arcsec})$. 
Table 2. Basic parameters of the Antlia galaxy sample: (1) ID from FS90, (2)-(3) J2000 coordinates, (4) morphology from FS90, (5) Galactic extinction from Schlafly \& Finkbeiner (2011), (6)-(12) global properties calculated in this work (Sérsic index, central surface brightness, scale radius, effective surface brightness, effective radius, $T_{1}$-band magnitude, $\left(C-T_{1}\right)$ colour), (13) radial velocity. The full table can be accessed electronically.

\begin{tabular}{|c|c|c|c|c|c|c|c|c|c|c|c|c|}
\hline $\begin{array}{l}(1) \\
\text { FS90 } \\
\text { ID }\end{array}$ & $\begin{array}{c}(2) \\
\text { RA } \\
\text { J2000 }\end{array}$ & $\begin{array}{c}(3) \\
\text { Dec } \\
\text { J2000 }\end{array}$ & $\begin{array}{c}(4) \\
\text { FS90 } \\
\text { morph. }\end{array}$ & $\begin{array}{c}(5) \\
E(B-V) \\
\text { mag }\end{array}$ & $\begin{array}{l}(6) \\
\mathrm{n} \\
-\end{array}$ & $\begin{array}{c}(7) \\
\mu_{0} \\
\operatorname{mag} \operatorname{arcsec}^{-2}\end{array}$ & $\begin{array}{c}(8) \\
r_{0} \\
\operatorname{arcsec}\end{array}$ & $\begin{array}{c}(9) \\
\mu_{\mathrm{e}} \\
\operatorname{mag}_{\operatorname{arcsec}}{ }^{-2}\end{array}$ & $\begin{array}{c}(10) \\
r_{\mathrm{e}} \\
\operatorname{arcsec}\end{array}$ & $\begin{array}{c}(11) \\
T_{1} \\
\text { mag }\end{array}$ & $\begin{array}{c}(12) \\
\left(C-T_{1}\right) \\
\operatorname{mag}\end{array}$ & $\begin{array}{c}(13) \\
v_{r} \\
\mathrm{~km} \mathrm{seg}^{-1}\end{array}$ \\
\hline 72 & $10: 29: 20$ & $-35: 38: 24$ & So & 0.067 & 1.58 & 18.11 & 1.19 & 21.18 & 6.14 & 14.33 & 1.93 & $2986 \pm 38^{b}$ \\
\hline 73 & $10: 28: 10$ & $-35: 42: 55$ & $\mathrm{dE}$ & 0.065 & 1.28 & 20.54 & 1.57 & 22.96 & 4.38 & 16.95 & 1.69 & ... \\
\hline 78 & $10: 28: 16$ & $-35: 46: 24$ & $\mathrm{dE}$ & 0.067 & 0.78 & 23.71 & 4.42 & 25.07 & 5.27 & 18.88 & 1.68 & $\cdots$ \\
\hline 79 & $10: 28: 19$ & $-35: 27: 16$ & So & 0.073 & 1.86 & 16.73 & 0.71 & 20.43 & 6.98 & 13.21 & 2.12 & $2734 \pm 36^{b}$ \\
\hline 84 & $10: 28: 23$ & $-35: 31: 46$ & E & 0.073 & 1.89 & 16.65 & 0.53 & 20.39 & 5.50 & 13.69 & 2.05 & $2428 \pm 30^{b}$ \\
\hline 85 & $10: 28: 24$ & $-35: 34: 21$ & $\mathrm{dE}$ & 0.072 & 0.61 & 23.22 & 4.44 & 24.20 & 4.18 & 18.62 & 1.68 & $2000 \pm 200^{a}$ \\
\hline 94 & $10: 28: 31$ & $-35: 42: 18$ & So & 0.065 & 2.60 & 13.72 & 0.06 & 19.01 & 3.37 & 13.20 & 2.01 & $2786 \pm 45^{b}$ \\
\hline 103 & $10: 28: 45$ & $-35: 34: 38$ & $\mathrm{dE}$ & 0.075 & 2.46 & 20.75 & 0.11 & 25.73 & 4.83 & 19.18 & 1.98 & $2092 \pm 29^{b}$ \\
\hline
\end{tabular}

Notes. - Radial velocities are from: ${ }^{a}=$ Smith Castelli et al. (2012), ${ }^{b}=\mathrm{NED}^{1},{ }^{c}=$ Caso \& Richtler (2015).

${ }^{1}$ This research has made use of the NASA/IPAC Extragalactic Database (NED) which is operated by the Jet Propulsion Laboratory, California Institute of Technology, under contract with the National Aeronautics and Space Administration.

\subsection{Numerical fits to the surface brightness profiles}

To fit the brightness profiles, we used a uni-dimensional Sérsic model (Sérsic 1968), which can be expressed as follows:

$I(r)=I_{\mathrm{e}} \mathrm{e}^{-b_{n}\left[\left(\frac{r}{r_{\mathrm{e}}}\right)^{1 / n}-1\right],}$

while in magnitudes per square aresec it is:

$\mu(r)=\mu_{\mathrm{e}}+1.0857 b_{n}\left[\left(\frac{r}{r_{\mathrm{e}}}\right)^{1 / n}-1\right]$,

where $r_{\mathrm{e}}$ is the effective radius, $\mu_{\mathrm{e}}$ is the effective surface brightness, and $n$ is the Sérsic index, which is a measure of the concentration of the profile. The constant $b_{n}$ depends on the shape parameter $n$ and is obtained numerically by solving the following equation (Ciotti 1991),

$\frac{\Gamma(2 n)}{2}=\gamma\left(2 n, b_{n}\right)$,

where $\Gamma(x)$ is the complete gamma function and $\gamma(a, x)$ the incomplete gamma function. An alternative way to express the Sérsic model, in terms of intensity, is the following:

$I(r)=I_{0} \mathrm{e}^{-\left(\frac{r}{r_{0}}\right)^{N}}$,

where $I_{0}$ is the central surface brightness, $r_{0}$ is a scale parameter, and $N=1 / n$. If we express the above equation in units of magnitude per square arcsec:

$\mu(r)=\mu_{0}+1.0857\left(\frac{r}{r_{0}}\right)^{N} ;$

which is the one used for our profile fits, where $\mu_{0}$ is the central surface brightness. The transformation between effective radius and scale parameter can be obtained using equations 4 and 8 :

$I_{0} \mathrm{e}^{-\left(\frac{r}{r_{0}}\right)^{N}}=I_{\mathrm{e}} \mathrm{e}^{-b_{n}\left[\left(\frac{r}{r_{\mathrm{e}}}\right)^{1 / n}-1\right]}=\mathrm{e}^{b_{n}} I_{\mathrm{e}} \mathrm{e}^{-b_{n}\left(\frac{r}{r_{\mathrm{e}}}\right)^{1 / n}}$.

Considering $r=0$, we obtain

$I_{0}=\mathrm{e}^{b_{n}} I_{\mathrm{e}}$

and then, $r_{0}=b_{n}^{-n} r_{\mathrm{e}}$.
The total flux is obtained by solving the integral:

$F_{\mathrm{T}}=\int_{0}^{\infty} 2 \pi I(r) r \mathrm{~d} r=2 \pi \int_{0}^{\infty} \mathrm{e}^{-b_{n}\left[\left(\frac{r}{r_{\mathrm{e}}}\right)^{1 / n}-1\right]} r \mathrm{~d} r$,

which leads us to:

$F_{\mathrm{T}}=2 \pi r_{\mathrm{e}}^{2} b_{n}^{-2 n} I_{\mathrm{e}} n \mathrm{e}^{b_{n}} n \Gamma(2 n)$.

The integral magnitude is obtained by transforming the above equation,

$m=C_{0}-2.5 \log \left(2 \pi r_{\mathrm{e}}^{2} b_{n}^{-2 n} I_{\mathrm{e}} n \mathrm{e}^{b_{n}} n \Gamma(2 n)\right)$

$m=\mu_{0}-1.99-5 \log \left(r_{\mathrm{e}}\right)+5 n \log \left(b_{n}\right)$

$$
-2.5 \log (n \Gamma(2 n)) \text {. }
$$

The fits were obtained using the task NFIT1D from IRAF, which implements the $\chi^{2}$ statistic test through the Levenberg-Marquardt algorithm. We excluded the inner arcsec of the profile in the fits, in order to minimize seeing effects. We will show in the next subsection that, in this way, the fits are not significantly affected by seeing for galaxies with $n \lesssim 3$. In most cases, we have been able to fit the profiles with a single Sérsic model obtaining residuals smaller than $0.5 \mathrm{mag}$. We want to remark that the scale parameters presented in this paper have been derived without trying a bulge-disc profile decomposition. Table 2 shows some of the scaling parameters and photometric magnitudes obtained for the sample.

\subsection{Effects of seeing on the modelled parameters}

Ground-based images are affected by atmospheric seeing; for images of extended objects it always acts distributing light from higherto lower-surface brightness regions, thus mainly affecting the central portions of early-type galaxies profiles.

Instead of modelling out seeing effects on the fitted parameters (Trujillo et al. 2001a,b), we performed a series of simple simulations of artificial galaxies following the procedure explained by Gavazzi et al. (2005). Using the task MKOBJECTS from IRAF ARTDATA package, we built a series of FITS images of simulated galaxies with Sérsic light profiles and null ellipticity. In addition, we fixed $\mu_{0}$ in $10 \mathrm{mag} \operatorname{arcsec}^{-2}$, while the Sérsic index ranged between 0.5 and 4 . Finally, we added to each simulated image a sky level and noise similar to those on the real images. 


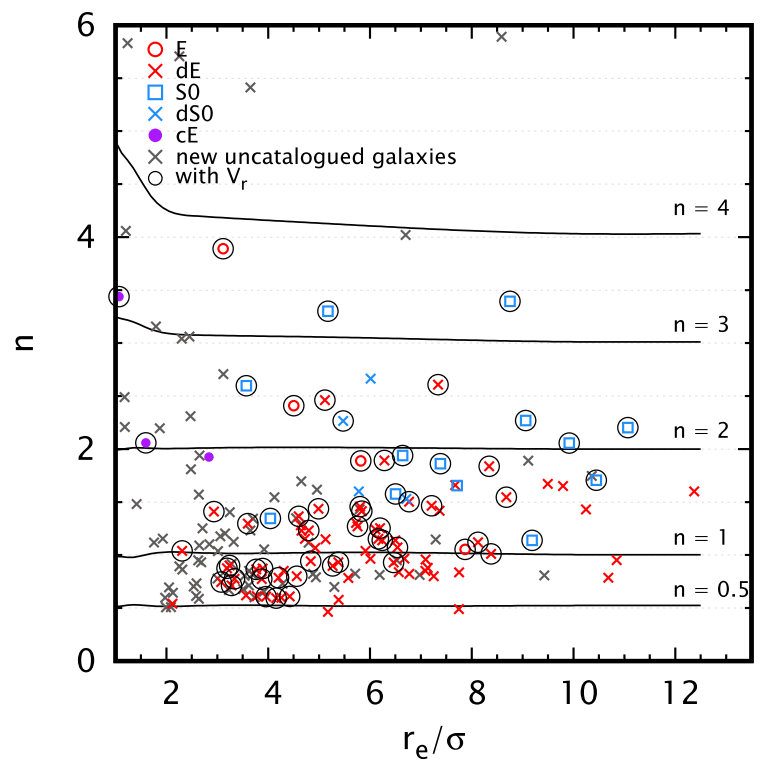

Figure 3. Measured Sérsic index $n$ versus effective radius $r_{\mathrm{e}}$ (in units of the PSF dispersion). The solid lines show the results for artificial galaxies and the symbols for real ones.

To simulate the seeing effects, we performed a convolution using the GAUSS task, with Gaussian profiles and $\sigma(\sigma=0.42466$ FWHM $)$ in the range of $0.5-10$ arcsec. The Sérsic model was fitted to the simulated galaxies following exactly the same procedure as for the real galaxies, excluding the innermost arcsec from the profile.

Fig. 3 shows the results obtained for Sérsic index $n$ versus $r_{\mathrm{e}} / \sigma$. The symbols indicate different galaxy morphologies and the lines correspond to different theoretical (model) Sérsic indices. It can be seen that for small $n$ values, the Sérsic indices measured from the convolved fake galaxies follow reasonably well their theoretical values; however, for $n>3$ there are significant differences for small $r_{\mathrm{e}} / \sigma$, in the sense that the measured $n$ is overestimated. This result is in agreement with that obtained by Gavazzi et al. (2005), and it is due to light being distributed off the galaxy centre by seeing, thus leading to a measured Sérsic index that is higher than the intrinsic one. This effect is of course stronger for more concentrated (i.e. $n>3$ ) profiles.

Given that there are very few real galaxies in the sample within the ranges of $n$ and $r_{\mathrm{e}}$ where the effect of seeing is significant, it was decided not to perform a general correction for seeing.

\subsection{Signal-to-noise ratio}

In order to estimate the quality of the profile fits and the parameters obtained, we calculated how the $\mathrm{S} / \mathrm{N}$ varies as a function of the equivalent radius of the profile using the following expression (McDonald et al. 2011):

$$
\frac{S}{N}(r)=\frac{I_{\mathrm{t}}(r)\left[\text { pixel }^{-2}\right] \sqrt{A_{\text {iso }}}[\text { pixel }]}{\sqrt{I_{\mathrm{s}}}\left[\text { pixel }^{-1}\right]},
$$

where $A_{\text {iso }}$ is the area of the given isophote in pixels ${ }^{2}$,

$A_{\text {iso }}=2 \pi \sqrt{0.5\left(a^{2}+b^{2}\right)}$,

where $a$ and $b$ are the semi-axes (major and minor) of the elliptic isophote, $I_{\mathrm{t}}(r)$ the total surface brightness of the galaxy, and $I_{\mathrm{s}}$ the sky surface brightness. The $\mathrm{S} / \mathrm{N}$ for the fainter galaxies in the present sample $\left(T_{1}>14 \mathrm{mag}\right)$ at the isophote $27.5 \mathrm{mag} \operatorname{arcsec}^{-2}$ ranges between $1.6 \pm 0.3$ and $3.0 \pm 1.0$.

\section{RESULTS}

\subsection{Comparison between isophotal and model-fit effective radii}

The effective radius may be measured in different ways. In our case, we obtained it by fitting a single Sérsic model to the observed profile so that $r_{\mathrm{e}}$ encloses half the luminosity of the model integrated to infinity (Calderón et al. 2015). Now, we want to compare these effective radii with the ones calculated directly from the isophotal parameters corresponding to $\sim 27 \mathrm{mag} \mathrm{arcsec}^{-2}$ in the $T_{1}$ band. Fig. 4(a) shows the difference between the $r_{\mathrm{e}}$ calculated by Calderón et al. (2015) performing an extrapolation to infinity and the 'isophotal' ones, versus absolute (top axis) and apparent (bottom axis) $T_{1}$ magnitudes. At the bottom of the same figure, we include a histogram that shows the number of galaxies considered in each magnitude bin, depicted on the right axis. The total galaxy sample is represented in green, candidate members in light grey, members in red, and members with measured radial velocity in black.

It is important to remark that for the two brightest galaxies $\left(M_{\mathrm{T}_{1}}<-22 \mathrm{mag}\right)$, the effective radius results underestimated when using a single component profile (for NGC 3268 the difference is even larger than $1 \mathrm{kpc}$ ). A similar (although milder) tendency is present for S0s and cEs. It can be seen that, as expected, the confirmed dEs show mostly positive differences, while the new galaxies (mainly candidates) are the ones showing more negative differences. This effect is less noticeable if we consider a similar difference but for the effective surface brightness (Fig. 4b). In this case, the confirmed dEs are evenly distributed about zero.

\subsection{Geometrical parameters at different galactocentric radii}

In this section, we analyse the geometrical parameters obtained from the ELLIPSE output for the whole sample, considering the four radial ranges (regions 1 to 4 ) defined in Section 4. Fig. 5 shows the distribution of the intensity-weighted average ellipticity $\langle\epsilon\rangle$ for the four regions, with a cross-hatched (red) histogram for faint galaxies (dEs and $\mathrm{dSph}$ ) and an open one for the whole sample. We note that the morphological classification was done by visual inspection of each galaxy, following the criteria used in FS90. That is why we do not establish a magnitude limit (usually set around $M_{B}=-18 \mathrm{mag}$ ); an overlap in luminosity between bright and dwarf ellipticals can thus be seen.

The histograms of mean ellipticity show flatter (although slightly less extended) distributions, as compared to those obtained by Chaware et al. (2014) and Hao et al. (2006), where a main peak at $\langle\epsilon\rangle \sim 0.1 \rightarrow 0.16$ is evident in regions 1 to 4 , implying a dominant fraction of nearly round galaxies. Besides a similar low $\langle\epsilon\rangle$ peak, a second peak at $\langle\epsilon\rangle \approx 0.3$ is also evident in region 1 of our sample. This reflects the fact that most of the brighter galaxies in Antlia are lenticulars (S0), while dwarfs also tend to exhibit relatively large flattenings, despite most of them being classified as $\mathrm{dE}$ (dS0s are found only among the brighter dwarfs.).

Figs 6 and 7 show the distributions of the weighted mean values of the Fourier coefficients $\left\langle a_{3} / a\right\rangle$ and $\left\langle a_{4} / a\right\rangle$. As usual, they are reasonably fitted with a single Gaussian centred at zero, except in the outermost region, where the distribution is much flatter. In particular, the coefficient $\left\langle a_{4} / a\right\rangle$ is slightly positive in regions 1 through 3 for the dwarf galaxies, which indicates an excess of discy 


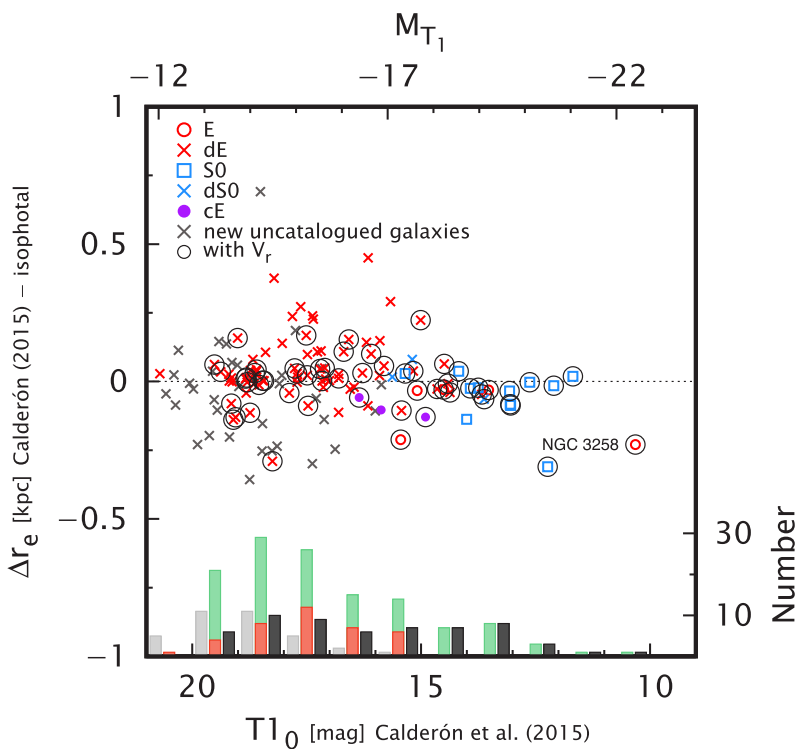

(a)

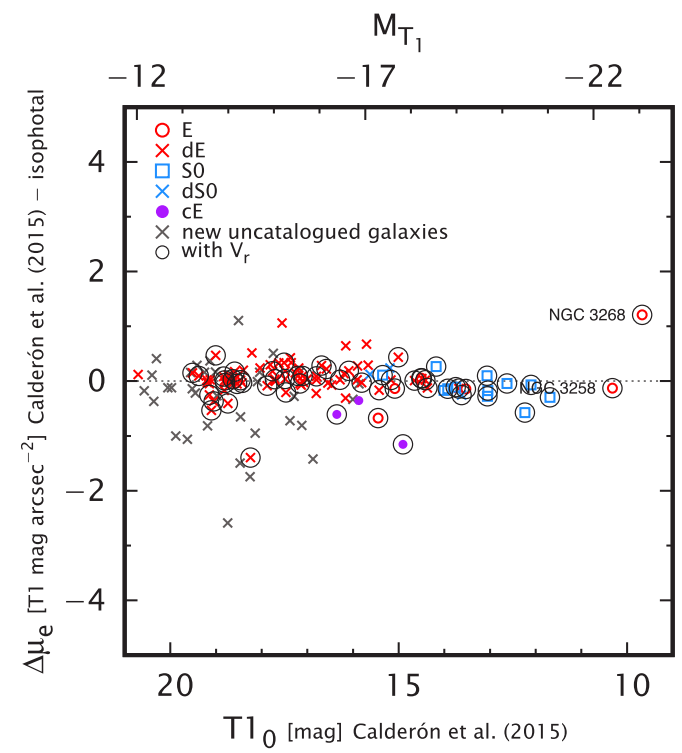

(b)

Figure 4. Differences between structural parameters obtained from Sérsic fits and isophotal ones. (a) Difference between $r_{\mathrm{e}}$ calculated by Calderón et al. (2015) fitting a Sérsic model and the 'isophotal' $\sim 27 \mathrm{mag} \mathrm{arcsec}^{-2}$ ones, versus absolute and apparent $T_{1}$ magnitudes (top and bottom axes), respectively. (b) Same as plot (a) but for the difference in effective surface brightness.

isophotes. On the contrary, the brighter galaxies show an excess of negative values in region 2, pointing to boxy isophotes. Fig. 6 shows, for the brighter galaxies, an excess of negative values of the $\left\langle a_{3} / a\right\rangle$ coefficient in the innermost region; this can be related with minor mergers (Ryden et al. 2001).

In order to assess the significance of any differences between the weighted-mean values along the equivalent radius, we perform a two-sample Kolmogorov-Smirnov (KS) test between adjacent regions (Press et al. 1992), considering the whole sample. Regarding the mean ellipticity (Fig. 8), the KS test shows that adjacent regions may share the same distribution (see Table 3). The two-peaked distribution for region 1, although visually evident in Fig. 5, is thus

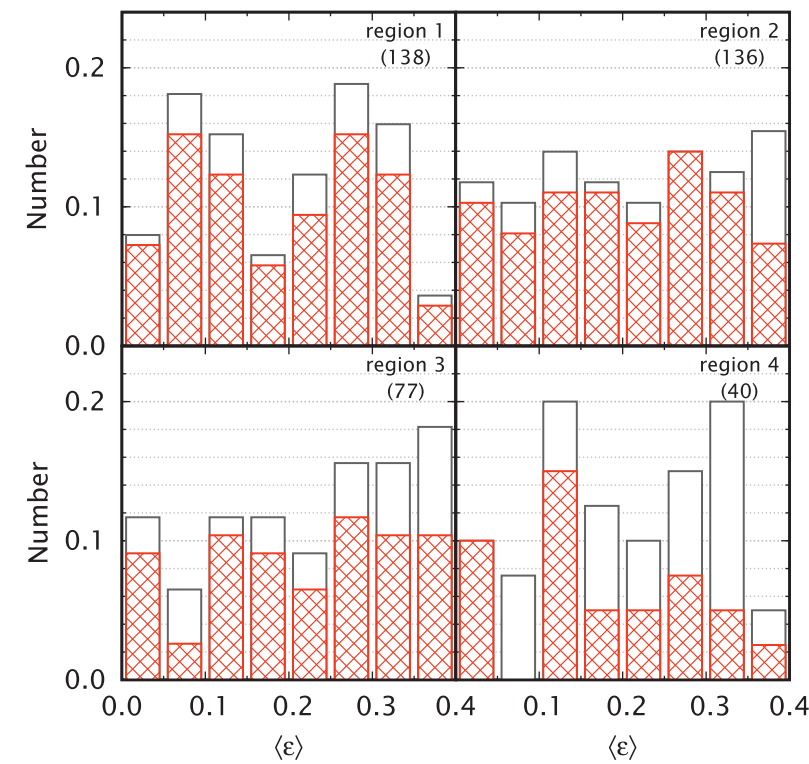

Figure 5. Histograms of the mean weighted ellipticity distributions, crosshatched in red for $\mathrm{dE}$ and $\mathrm{dSph}$ and open for the whole sample. Number of galaxies in each region is indicated in parentheses.

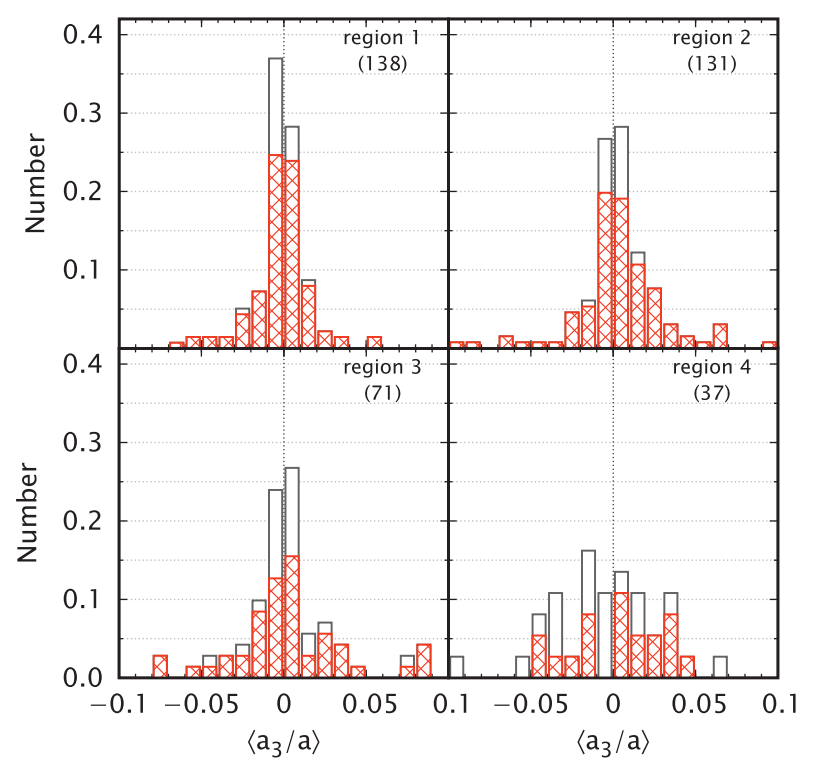

Figure 6. Same as Fig. 5 but for the $\left\langle a_{3} / a\right\rangle$ parameter distributions.

not significantly different (at a 5 per cent significance level) from the distributions in the other regions. With this in mind, we plot separately with red circles the fainter galaxies of the sample and with back squares the brighter sample, to compare their respective behaviours. While dwarfs seem to be mostly responsible for the disappearance of the $\langle\epsilon\rangle \approx 0.1$ peak when going from region 1 to region 2 , brighter galaxies play this role for the $\langle\epsilon\rangle \approx 0.3$ peak. A qualitative analysis of Fig. 8, then, shows that dwarfs on the low- $\epsilon$ peak in region 1 have been shifted to both higher and lower ellipticities in region 2, while bright galaxies on the high- $\epsilon$ peak have been mostly shifted to still higher ellipticities. This means that some of the brighter galaxies display positive ellipticity gradients (consistent with a S0 morphology), while dwarf galaxies may display either positive or negative gradients. 


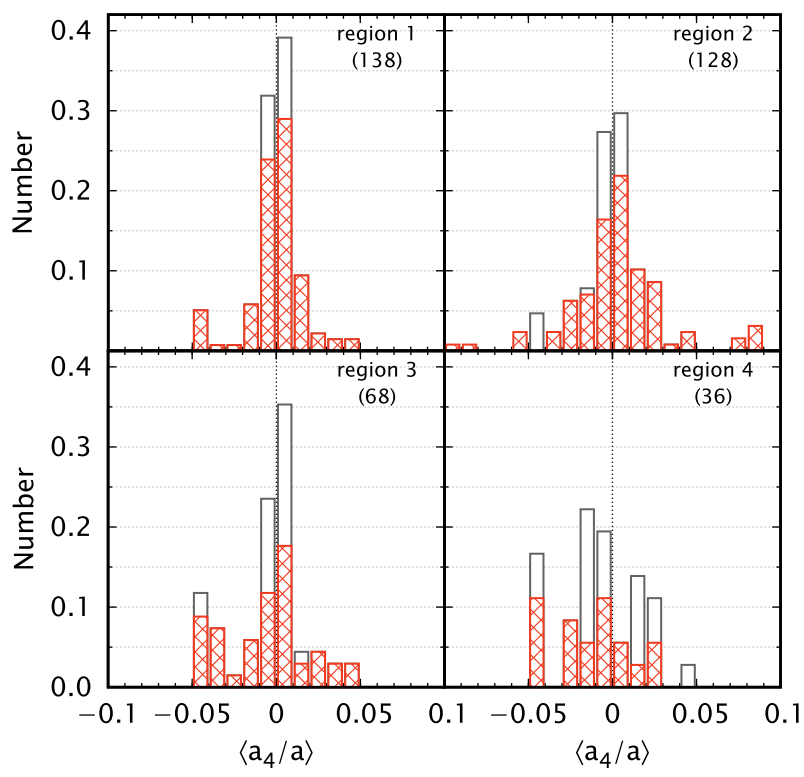

Figure 7. Same as Fig. 5 but for the $\left\langle a_{4} / a\right\rangle$ parameter distributions.

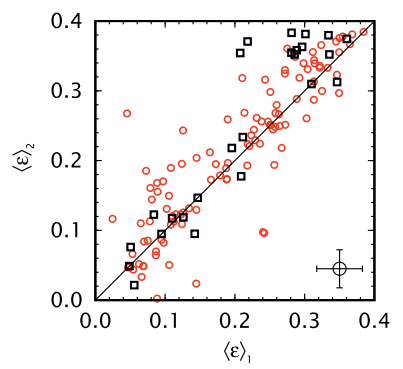

(a) Region 2 versus region 1 .

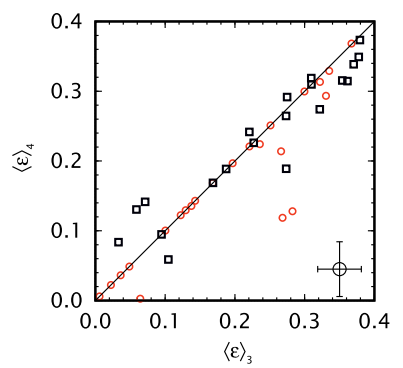

(c) Region 4 versus region 3.

Figure 8. Comparison of $\langle\epsilon\rangle$ values between adjacent regions. The open circle at the lower right corner shows the median error bars for each panel.

Table 3. Results from the two-sample KS test $(D)$ for the mean ellipticity. The probability to support the hypothesis that the compared distributions are taken from the same parent distribution is given by $P$.

\begin{tabular}{lcccccc}
\hline & \multicolumn{2}{c}{ Region 2 } & \multicolumn{2}{c}{ Region 3 } & \multicolumn{2}{c}{ Region 4 } \\
& $D$ & $P$ & $D$ & $P$ & $D$ & $P$ \\
\hline Region 1 & 0.125 & 0.222 & 0.166 & 0.118 & 0.130 & 0.634 \\
Region 2 & & & 0.108 & 0.579 & 0.120 & 0.732 \\
Region 3 & & & & & 0.148 & 0.570 \\
\hline
\end{tabular}

\subsection{Relations between $\left\langle a_{4} / a\right\rangle$ and $\langle\epsilon\rangle$, Sérsic index and luminosity}

Fig. 9(a) shows the relation between $\left\langle a_{4} / a\right\rangle$ and ellipticity (on each region). As already shown in Fig. 7, we see that the $\left\langle a_{4} / a\right\rangle$ distributions get broader for larger radii (regions 1 to 4 ), with a slight excess of positive $\left\langle a_{4} / a\right\rangle$ values in the first two regions, indicating a dominance of discy isophotes. These features are evident along the full range of ellipticities, so there is no trend of $\left\langle a_{4} / a\right\rangle$ with $\langle\epsilon\rangle$. Fig. 9(b) shows no clear correlation between $\left\langle a_{4} / a\right\rangle$ and Sérsic index. There is a group of galaxies with negative $\left\langle a_{4} / a\right\rangle$ and low $n$ in region 1 , but the tendency is washed out in the outermost regions. Finally, Fig. 9(c) clearly shows that the dispersion in the $\left\langle a_{4} / a\right\rangle$ distribution increases with decreasing galaxy luminosity, with an important increment for galaxies with $M_{\mathrm{T}_{1}}>-16 \mathrm{mag}$. The tendency in region 1 is not clear; however, in regions 2 and 3 there are more galaxies (particularly dwarfs) with discy isophotes. As in the other plots, the scatter of the relation increases rapidly as we go through region 1 to region 4 . These plots are in agreement with Chaware et al. (2014), extending the range of surface brightnesses at the faint end.

We applied Spearman's rank correlation $(\rho)$ test (Spearman 1904), which is used to decide whether a pair of variables are correlated or not, to the data of Fig. 9. Its advantages over the Pearson correlation test are that it is non-parametric, and a linear relationship between the variables is not a requirement. When we consider the complete sample, the test results in small values for the $\left\langle a_{4} / a\right\rangle-n$ relation in regions 1 and 4 , which implies a high correlation probability between both variables. The Spearman coefficients are $\rho=0.09$ and $\rho=0.17$, respectively, which lead to probabilities $p=0.24$ and $p=0.28$ that the null hypothesis (i.e. no correlation) is true. Almost the same happens if we just consider the $\mathrm{dE}$ and $\mathrm{dSph}$ galaxies. For the relation $\left\langle a_{4} / a\right\rangle$ versus $\langle\epsilon\rangle$ the picture is similar, although just considering regions 2 and 4 . The correlation coefficient values given by the test are: $\rho=-0.2$ for region 2 , and $\rho=0.4$ for region 4; the probability of the null hypothesis (no correlation) being true is $p=0.01$ for both regions.

\section{DISCUSSION}

The study of the distributions of isophotal parameters in different ranges along the radial profiles of the galaxies is used as a tool to look for statistical differences between inner and outer parts of the galaxies, and their possible correlations with global galaxy properties. In this section, we compare our results with numerical simulations that involve galaxy mergers that took place out of any deep gravitational potential, such as a cluster. Thus, it should be taken into account that repeated tidal interactions may further affect the structural properties of cluster galaxies. Also, the merges themselves can be modified by the cluster potential well.

The ellipticity distribution (Fig. 5) in our sample shows a main peak around $\langle\epsilon\rangle \sim 0.28$, and a second peak around $\langle\epsilon\rangle \sim 0.08$; this makes the galaxies in the Antlia cluster more flattened in comparison to the samples presented in Chaware et al. (2014) and Hao et al. (2006). These differences may be explained by the distinctive characteristics of the galaxy sample, the Antlia one being dominated by lenticular galaxies. The shapes of the ellipticity histograms in regions 1 and 4 are similar, showing two peaks around the same mean ellipticities. This still holds when we consider the full range of radii. The dEs, which are shown in red, follow the same trend as all the other morphologies; this is true for all regions, except for region 4, which shows a large fraction of rounder dEs. This behaviour is also 


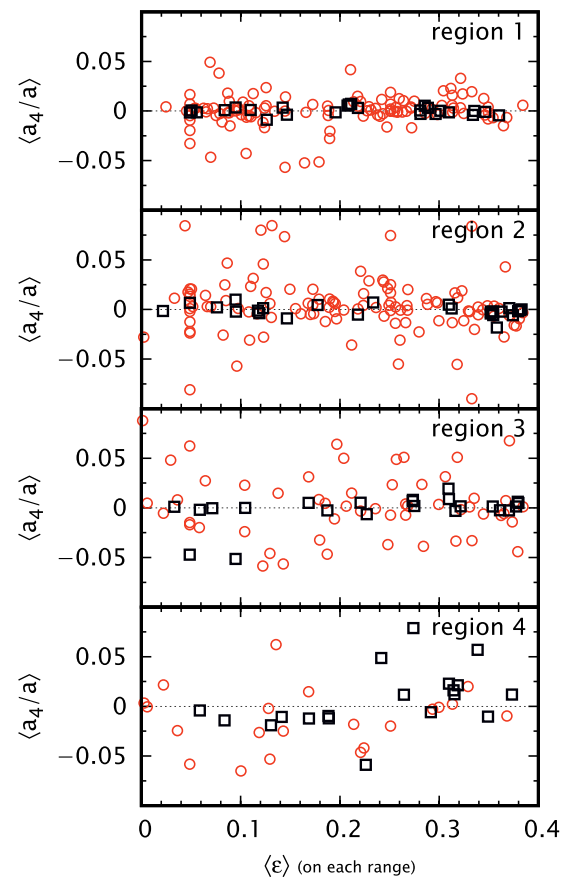

(a)

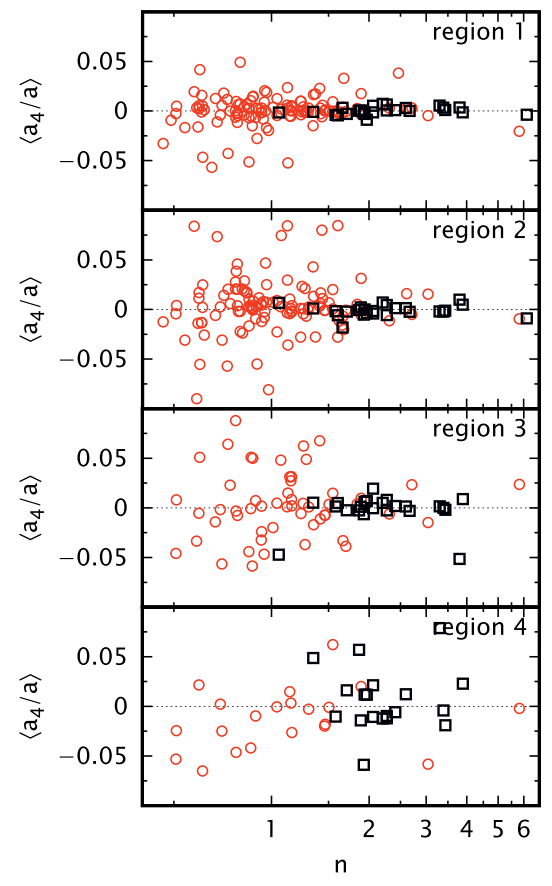

(b)

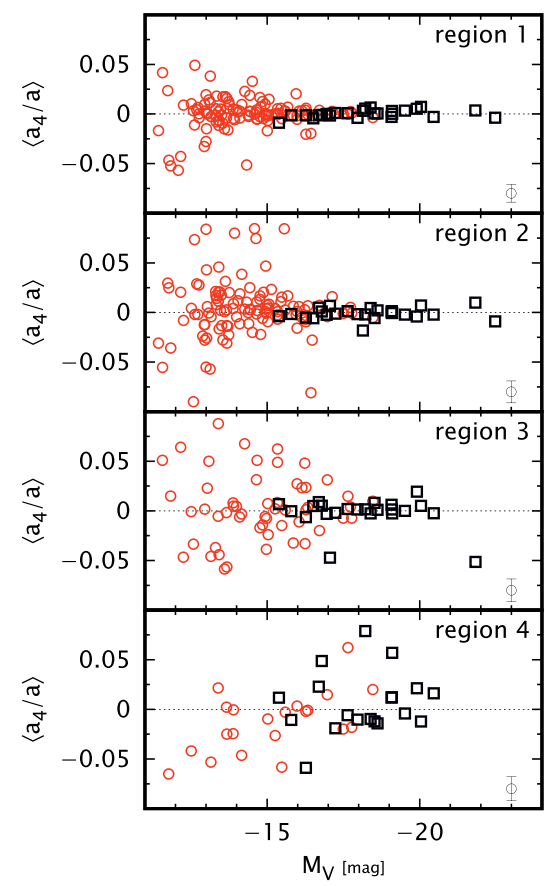

(c)

Figure 9. Relation between $\left\langle a_{4} / a\right\rangle$ and (a) $\langle\epsilon\rangle$, (b) $n$, and (c) luminosity. Each panel, on each figure, represents regions 1 to 4 , from top to bottom. We identify $\mathrm{dE}$ and dSph galaxies of the sample with red circles and the brighter ones with black squares. The error bars on the last figure are as in Fig. 8 .

found in hydrodynamic simulations (Tenneti et al. 2015). The KS test, however, shows no statistical differences between the ellipticity distributions for the inner and outer regions of the galaxies in the sample (Table 3), so the above mentioned differences should be regarded as marginal.

The deviations from perfect ellipses, measured by the Fourier coefficients, have been studied since Lauer (1985). However, several issues are pending and a new discussion is still relevant. The $a_{4}$ coefficient is an intrinsic parameter of the galaxy, without (projection) dependence on the viewing angle. Khochfar \& Burkert (2005) used $\mathrm{N}$-body simulations to predict that the percentage of discyboxy galaxies is affected by the environment, so that in overdense regions, galaxies with more discy shape isophotes are produced (see also Pasquali et al. 2006).

The separation in radial bins of $\left\langle a_{4} / a\right\rangle$ shows that, for our sample, the distributions of the two innermost regions are similar to each other, and the KS test does not reveal any statistical difference between them. The percentage of discy isophotes is larger in all regions, except in region 4 . There appears not to be a strong correlation between $\left\langle a_{4} / a\right\rangle$ and ellipticity, in agreement with Hao et al. (2006) and Chaware et al. (2014). The peculiar distribution of $\left\langle a_{4} / a\right\rangle$ in region 4 may be the result of the intrinsic merger history of the cluster. $N$-body simulations by Bournaud, Jog \& Combes (2005), which take into account mergers of galaxies with different mass ratios (outside a cluster gravitational potential), produce galaxies with boxy isophotes in the inner part of the profile and discy ones in the outer part (i.e. positive radial gradients in $\left\langle a_{4} / a\right\rangle$ ). Considering the sample studied in this work, the profiles do not clearly show this behaviour, with half of them showing negative gradients for the mean $\left\langle a_{4} / a\right\rangle$. The Antlia sample has a mild predominance of galaxies with $\left\langle a_{4} / a\right\rangle>0$ in the innermost regions: 55 per cent for region 1,54 percent for region 2 (most are in the range of $0.0-0.02$ ). This was pointed out by Naab \& Burkert (2003) as the result of binary disc galaxy mergers, from collisionless $N$-body simulations. The larger values of $\left\langle a_{4} / a\right\rangle$ may be related with hybrid mergers with very different mass ratios (Bournaud et al. 2005).

As pointed out by Calderón et al. (2015), the CMR of the sample shows a 'break' at the bright end so that the most massive ETGs show almost constant colours. One possible interpretation is that this is a consequence of dry mergers - both minor and major - since $z \sim 2$. Then, the more massive galaxies would evolve without gas and no further enrichment is expected (Jiménez et al. 2011). The analysis of geometrical parameters may show evidence of different possible scenarios. The largest galaxies in the sample have regular isophotes $\left(\left\langle a_{4} / a\right\rangle \sim 0\right)$ and the ellipticities show a wide distribution along the range: $0.0-0.4$, while dEs have large deviations from perfect isophotes. Numerical simulations of multiple mergers presented by Bournaud, Jog \& Combes (2007) show that the merger remnants tend to be boxy for 1:1 mergers (see also Naab \& Burkert 2003; Naab \& Trujillo 2006, for dissipationless simulations), while larger mass ratio (like $3: 1$ and 4:1) mergers result in discy-shape ellipticals. Pasquali et al. (2006, and references therein) found that discy galaxies have higher ellipticities in the sample that they studied. On the other hand, boxy galaxies have larger half-light radii, and tend to be bigger and brighter than discy galaxies. Chaware et al. (2014) and He et al. (2014) (also reported by Ferrarese et al. 2006) found that their sample shows a trend between $a_{4} / a$ and absolute magnitude in the $i$ band, which could be considered similar to the boxiness trend found by Bournaud et al. (2007) for the remnants of multiple minor mergers, with boxiness increasing with mass ratio. In particular, we could not confirm any relation between $\left\langle a_{4} / a\right\rangle$ and magnitude in our sample. In any case, it is clear that early-type dwarfs display a broad range in $\left\langle a_{4} / a\right\rangle$ at all radii, from fairly discy to boxy shapes. This could be due to dwarfs being more strongly affected by interactions, and/or to a mixture of objects with different origins/histories among low-luminosity systems. 
The relations between the Sérsic index $n$ and $a_{4} / a, a_{3} / a$ and $\epsilon$ have been studied by different authors on different magnitude ranges (Hao et al. 2006; He et al. 2014), who found only a mild correlation among $n, \epsilon$, and $a_{4} / a$. We found a correlation between these parameters just for the innermost radial range; this behaviour still holds when we only consider the faintest galaxies in the sample. We also point out that the relatively broad ranges spanned by the values of the Fourier parameters of dEs cannot be explained just by the larger errors present in the relations depicted in Fig. 9. Thus, it may be an intrinsic characteristic for the fainter galaxies, which has been shown to include several structural sub-classes pointing to different origins (Cellone \& Buzzoni 2005; Lisker et al. 2007, and references therein).

\section{SUMMARY}

We present the isophotal analysis as well as the surface photometry data (catalogue) for a sample of 138 early-type galaxies in the Antlia cluster. The scaling relations followed by them have been described in a previous companion paper (Calderón et al. 2015). Our study is based on MOSAIC II-CTIO images of four adjoining and slightly superimposed fields, covering each one $36 \times 36 \mathrm{arcmin}^{2}$, and taken with the Kron-Cousins $R$ and Washington $C$ filters.

We have used ELLIPSE within IRAF to obtain the geometrical parameters that characterize the isophotes of each galaxy along its radius. Then, we obtained mean values of ellipticity and Fourier coefficients $a_{3}$ and $a_{4}$ in four radial bins, weighted by the intensity of each isophote. Total integrated magnitudes were obtained by fitting single Sérsic models to the observed surface brightness profiles. In addition to presenting the surface-photometry catalogue, our main goal was to find possible correlations among global properties. We also looked for statistical differences between the isophotal shapes in the inner and outer regions of the profiles, since it is supposed that physical processes ruling the evolution of galaxies affect both regions differently (Chaware et al. 2014, and references therein). Most of the galaxies in our sample have discy isophotes, but they tend to change along radius, turning into boxy. The processes involved in the evolution of the galaxies are presumably different: while in the inner part they must be driven by internal ones, the outer regions are more sensitive to the environment (ram-pressure stripping, galaxy harassment, etc.) as suggested by Kormendy \& Bender (2012).

\section{ACKNOWLEDGEMENTS}

We thank an anonymous referee for constructive remarks. This work was funded by grants from Consejo Nacional de Investigaciones Científicas y Técnicas de la República Argentina, Agencia Nacional de Promoción Científica y Tecnológica, and Universidad Nacional de La Plata (Argentina). JPC and LPB are grateful to the Departamento de Astronomía de la Universidad de Concepción (Chile) for financial support and warm hospitality during part of this research. MG acknowledges support from FONDECYT Regular Grant No. 1170121. LPB and MG: Visiting astronomers, Cerro Tololo Inter-American Observatory, National Optical Astronomy Observatories, which are operated by the Association of Universities for Research in Astronomy, under contract with the National Science Foundation.

\section{REFERENCES}

Barnes J. E., Hernquist L., 1992, ARA\&A, 30, 705

Bekki K., Shioya Y., 1997, ApJ, 478, L17
Bender R., Surma P., Doebereiner S., Moellenhoff C., Madejsky R., 1989, A\&A, 217, 35

Bertin E., Arnouts S., 1996, A\&AS, 117, 393

Bournaud F., Jog C. J., Combes F., 2005, A\&A, 437, 69

Bournaud F., Jog C. J., Combes F., 2007, A\&A, 476, 1179

Calderón J. P., Bassino L. P., Cellone S. A., Richtler T., Caso J. P., Gómez M., 2015, MNRAS, 451, 791

Canterna R., 1976, AJ, 81, 228

Caso J. P., Richtler T., 2015, A\&A, 584, A125

Cellone S. A., Buzzoni A., 2005, MNRAS, 356, 41

Chaware L., Cannon R., Kembhavi A. K., Mahabal A., Pandey S. K., 2014, ApJ, 787, 102

Ciotti L., 1991, A\&A, 249, 99

Dirsch B., Richtler T., Bassino L. P., 2003, A\&A, 408, 929

Emsellem E. et al., 2011, MNRAS, 414, 888

Ferguson H. C., Sandage A., 1990, AJ, 100, 1

Ferrarese L., van den Bosch F. C., Ford H. C., Jaffe W., O'Connell R. W., 1994, AJ, 108, 1598

Ferrarese L. et al., 2006, ApJS, 164, 334

Fukugita M., Shimasaku K., Ichikawa T., 1995, PASP, 107, 945

Gavazzi G., Donati A., Cucciati O., Sabatini S., Boselli A., Davies J., Zibetti S., 2005, A\&A, 430, 411

Geisler D., 1996, AJ, 111, 480

Hao C. N., Mao S., Deng Z. G., Xia X. Y., Wu H., 2006, MNRAS, 370, 1339

He Y.-Q., Hao C.-N., Xia X.-Y., 2014, Res. Astron. Astrophys., 14, 144

Jaffé Y. L., Aragón-Salamanca A., De Lucia G., Jablonka P., Rudnick G., Saglia R., Zaritsky D., 2011, MNRAS, 410, 280

Jedrzejewski R. I., 1987, MNRAS, 226, 747

Jiménez N., Cora S. A., Bassino L. P., Tecce T. E., Smith Castelli A. V., 2011, MNRAS, 417, 785

Khochfar S., Burkert A., 2005, MNRAS, 359, 1379

Kormendy J., Bender R., 2012, ApJS, 198, 2

Krajnović D. et al., 2013, MNRAS, 433, 2812

Lauer T. R., 1985, MNRAS, 216, 429

Lauer T. R. et al., 2005, AJ, 129, 2138

Lisker T., Grebel E. K., Binggeli B., Glatt K., 2007, ApJ, 660, 1186

Lisker T., Grebel E. K., Binggeli B., 2008, AJ, 135, 380

Malin D. F., Carter D., 1983, ApJ, 274, 534

McDonald M., Courteau S., Tully R. B., Roediger J., 2011, MNRAS, 414, 2055

Mei S. et al., 2012, Am. Astron. Soc. Meeting Abstr. 219, 411.06

Naab T., Burkert A., 2003, ApJ, 597, 893

Naab T., Trujillo I., 2006, MNRAS, 369, 625

Naab T., Khochfar S., Burkert A., 2006, ApJ, 636, L81

Nieto J.-L., Bender R., 1989, A\&A, 215, 266

Pasquali A. et al., 2006, ApJ, 636, 115

Penny S. J., Conselice C. J., 2008, MNRAS, 383, 247

Press W. H., Teukolsky S. A., Vetterling W. T., Flannery B. P., 1992, Numerical recipes in $\mathrm{C}$. The art of scientific computing. University Press, Cambridge

Rest A., van den Bosch F. C., Jaffe W., Tran H., Tsvetanov Z., Ford H. C., Davies J., Schafer J., 2001, AJ, 121, 2431

Ryden B. S., Forbes D. A., Terlevich A. I., 2001, MNRAS, 326, 1141

Schlafly E. F., Finkbeiner D. P., 2011, ApJ, 737, 103

Schweizer F., Seitzer P., 1988, ApJ, 328, 88

Seitzer P., Schweizer F., 1990, in Wielen R. ed., Dynamics and Interactions of Galaxies. Springer, Berlin, p. 270

Sérsic J. L., 1968, Atlas de galaxias australes. Observatorio Astronomico, Cordoba, Argentina

Smith Castelli A. V., Bassino L. P., Richtler T., Cellone S. A., Aruta C., Infante L., 2008a, MNRAS, 386, 2311

Smith Castelli A. V., Faifer F. R., Richtler T., Bassino L. P., 2008b, MNRAS, 391,685

Smith Castelli A. V., Cellone S. A., Faifer F. R., Bassino L. P., Richtler T., Romero G. A., Calderón J. P., Caso J. P., 2012, MNRAS, 419, 2472 
Spearman C., 1904, Am. J. Psychol., 15, 88

Statler T. S., 1994, AJ, 108, 111

Tenneti A., Mandelbaum R., Di Matteo T., Kiessling A., Khandai N., 2015, MNRAS, 453, 469

Trujillo I., Aguerri J. A. L., Cepa J., Gutiérrez C. M., 2001a, MNRAS, 321, 269

Trujillo I., Aguerri J. A. L., Cepa J., Gutiérrez C. M., 2001b, MNRAS, 328, 977

Valdes F., 1997, in Hunt G., Payne H., eds, IRAF data reduction software for the NOAO mosaic. ASP Conf. Ser. Vol. 125, Astronomical Data Analysis Software and Systems VI. Astron. Soc. Pac., San Francisco, p. 455

van den Bosch F. C., Ferrarese L., Jaffe W., Ford H. C., O’Connell R. W., 1994, AJ, 108, 1579

\section{SUPPORTING INFORMATION}

Supplementary data are available at MNRAS online.

\section{profiles.pdf \\ geometric-parameters.dat \\ full-catalogue.dat}

Please note: Oxford University Press is not responsible for the content or functionality of any supporting materials supplied by the authors. Any queries (other than missing material) should be directed to the corresponding author for the article.

This paper has been typeset from a $\mathrm{T}_{\mathrm{E}} \mathrm{X} / \mathrm{L} \mathrm{T} \mathrm{E} \mathrm{X}$ file prepared by the author. 